\title{
ESTRATÉGIA DE SERVIÇOS: O QUE HÁ DE COMUM ENTRE OS MODELOS TEÓRICOS?
}

\section{SERVICES STRATEGY: WHAT IS THERE IN COMMON AMONG THE THEORETICAL MODELS?}

\section{D0I: http://dx.doi.org/10.21714/raunp.v9i2.1633}

\author{
Mercia Cristiley Barreto \\ UERN. E-mail: profamerciac@gmail.com
}

\author{
Kleber Cavalcanti Nóbrega \\ Universidade Potiguar. E-mail: klebercn@unp.br
}

\author{
Lieda Amaral Souza \\ Universidade Potiguar. E-mail: lieda.amaral@unp.br
}

Envio em: Fevereiro de 2017

Aceite em: Agosto de 2017

\section{RESUMO}

Estratégia de serviços tem sido assunto recorrente entre autores como Teboul (1999), Hoffman (2009), Johnston (2002), Lovelock (2002), Zeithaml (2003), Corrêa (2009), Grönroos (2009), Nóbrega (2013), e Fitzsimmons \& Fitzsimmons, (2014). Até que ponto as proposições desses autores apontam convergências e distinções? Este foi o objetivo da presente pesquisa. A metodologia utilizada consistiu de revisão bibliográfica, com a identificação de similaridades e lacunas entre os modelos existentes. Foi desenvolvido um quadro, com a estruturação em dimensões enfocando os itens: conceituação, modelos e perspectivas, posicionamento, estratégia de preço e orientação para serviço. Foram apontadas as contribuições de cada autor em relação às dimensões, assim como registradas as lacunas. As similaridades que apresentaram mais congruências foram: conceituação, modelos e perspectivas e posicionamento. Por outro lado, as dimensões em que existem as maiores lacunas foram: estratégia de preço e orientação para servir. Foi possível identificar as principais contribuições entre os autores estudados, bem como, sugere-se recomendações para futuras pesquisas, bem como para a aplicação prática dessas teorias no âmbito empresarial.

Palavras-chaves: Estratégia de serviços. Gestão de serviços. Perspectivas estratégicas. Estratégia.

\section{ABSTRACT}

Service strategy has been recurrent subject matter among authors like Teboul (1999), Hoffman (2009), Johnston (2002), Lovelock(2002), ZeithamI (2003), Corrêa (2009), Grönroos (2009), Nóbrega (2013), and Fitzsimmons \& Fitzsimmons, (2014). To what extent do these authors propositions lead to convergences and distinctions? This was the objective of this research. The chosen methodology consisted of bibliographical review, with identification of similarities and gaps between the selected models. A framework was developed, structured on dimensions focusing the items: conceptualization, models and perspectives, positioning, price and service orientation. Each author's contribution was analyzed relatively to the six dimensions, as well as each gaps. The similarities with more congruence are: conceptualization, models and perspectives, and positioning. On the other hand, the dimensions with greatest gaps are: price and service orientation. It was possible to identify each author's main contributions, and recommendations are suggested for future research, as well as to practical aspects.

Key-words: Service strategy. Service management. Strategy. Strategic perspectives. 


\section{INTRODUÇÃO}

A evolução do mercado vem exigindo orientação para serviços cada vez mais qualificada. Isto é importante para as empresas do setor de serviços, bem como indústria, que almejam se destacar nos negócios. Organizações prestadoras de serviços procuram atender às necessidades de seus clientes, mas nem sempre os serviços são ofertados à altura das expectativas. Bhat (2012) O setor de serviços tem se tornado cada vez mais atuante no cenário mundial, por isso, as organizações necessitam reestruturar suas estratégias de serviços adotando novos posicionamentos em face desse tema.

As empresas buscam atender as necessidades do mercado existente através de bens e serviços altamente competitivos, entretanto, com a concorrência acirrada muitas organizações não conseguem obter atributos suficientemente diferenciados através das qualidades e estratégias de serviços (PARASURAMAN et al., 1994; ZEITHAML, 2011).

A estratégia de serviço define "de que forma o serviço pode ser incorporado à estratégia da organização, formulando a proposta de valor para o cliente alvo" (GRÖNROOS, 2009; NÓBREGA, 2013; FITZSIMMONS; FITZSIMMONS, 2014). Para tanto, adotar estratégia de serviços representa assumir riscos significativos perante a concorrência, onde o crescimento econômico é constituído pelas oportunidades desenvolvidas no País está reduzido em função da crise financeira instalada no mercado e no contexto estratégico global. Corroborando Sarkar (2008) afirma que estudar o setor de serviço é um desafio diário que se torna ainda maior quando se refere a estratégia de serviços com inovação.

Uma das dificuldades na teoria da estratégia de serviços, particularmente em relação à análise comparativa das estratégias de negócios, é a ausência metafórica de blocos de construção: descrições claras e consistentes de estratégias de negócios que são analisáveis a partir de diferentes perspectivas teóricas. Esta ausência apresenta uma lacuna no sentido acadêmico porque contribui para a fragmentação teórica do campo da estratégia de serviços. Os teóricos expressam as suas ideias por meio de modelos teóricos propostos, em termos que sejam significativos para aqueles que apresentam uma perspectiva teórica diferente (FULLER, 2010).

Entre 2013 e 2016, tem-se identificado um interesse significativo pela estratégia de serviços. Este fenômeno apresenta as condições extremamente relevantes para o mercado global, em que, por meio das oportunidades são identificadas as necessidades empresariais que desejam implementar estratégia de serviços. Para tanto, buscou-se estudar um setor que demonstrasse relevância para esta pesquisa, diante disso, foi apontado o segmento de serviços que conforme os dados estatísticos comprovados na Pesquisa Anual de Serviços (PAS) (2016) percebeu-se que os negócios relacionados ao setor de serviços crescem mundialmente, a qual mostra que este setor teve um crescimento de 2013 para 2016 de $76,2 \%$ da receita líquida de atividades consideradas de serviço e de $25,9 \%$ para serviços profissionais. O setor de serviços representa a maior parte do produto interno bruto do mundo (mais de 70\%) na maioria das economias estabelecidas (BITNER, 2014). São considerados de grande importância para o sucesso dos negócios e para o bem-estar da sociedade (BITNER, 2014). Além dos dados expostos, apresenta-se positivas contribuições econômicas no segmento de serviço, em que esta informação gera um aumento de interesse sobre esta temática, pelo fato, do setor de serviço ser considerado uma atividade econômica forte e de grande importância para o desenvolvimento social (GRÖNROOS, 2009). Corroborando Hoffman et al. (2016) os recursos observados nas organizações podem gerar vantagem competitiva por meio da prestação de serviços.

Por esta razão torna-se relevante investigar a relação dos principais modelos teóricos da estratégia de serviços. A motivação para estudar esse tema leva a crer que as organizações orientadas para servir apresentam necessidades em conhecer o mercado de atuação, pesquisam ferramentas para gestão baseadas nos recursos, com objetivo de agregar valor para seus bens ou serviços prestados (GRÖNROOS, 2009; NÓBREGA, 2013). Foi pensando nesse problema que surgiu o interesse em pesquisar os principais modelos teóricos acerca da estratégia de serviços. A importância dessa pesquisa para o meio empresarial e acadêmico é encontrar, dentre os modelos teóricos sobre estratégias de serviços, quais as contribuições comuns, as similaridades existentes, e, eventualmente, as lacunas dentre os modelos estudados. Diante disso, o objetivo desta pesquisa foi identificar interfaces, e eventualmente lacunas, entre os principais modelos de estratégia de serviços apresentados na literatura.

\section{CONCEPÇÃO TEÓRICA SOBRE ESTRATÉGIA DE SERVIÇOS}

As organizações apresentam produtos e serviços, por meio das suas estratégias de serviços desenvolvi- 
das, bem como, oferecem valor agregado ao cliente durante a prestação desses serviços. Segundo Nóbrega (2013) a estratégia de serviços estabelece como a organização indústria ou de serviço se propõe a oferecer serviços aos clientes, agregando valor ao seu produto principal, seja um bem ou serviço, para tanto, as estratégias genéricas mais utilizadas são: corporativa, competitiva e operações. A estratégia corporativa abrange toda a organização, compreendendo onde irá atuar, quando e quanto investir, bem como os níveis de retornos desejáveis. No entanto, a definição de estratégia competitiva que é o desdobramento da corporativa tem como objetivo definir a melhor forma para ser mais competitivo. A estratégia de operação visa garantir os processos de produção e entrega de valor ao cliente. Corroborando Golder et al. (2012), ao permitir que o cliente participe das etapas da prestação do serviço aumenta a probabilidade de ter maior satisfação e qualidade percebida dos bens ou serviços ofertados pelas organizações.

Segundo Grönroos (2009) são denominadas quatro perspectivas estratégicas importantes, 1 - Serviço: Caracteriza-se pelo desenvolvimento do pacote total de serviço, embora, a solução central esteja baseada em um produto físico, componentes do serviço prestado, informações complementares e atenção pessoal, são combinadas no pacote total de serviços. 2 - Produto Central: Baseia-se na solução do central (bens ou serviços), buscando prover valor tanto para o cliente como para usuário organizacional, onde a diferença da sua oferta dos outros, apresenta-se no fornecimento da solução central com excelência. 3 - Preço: A visão do preço é fator determinante de compra dos clientes, no entanto, manter o preço baixo é uma questão de sobrevivência da organização no mercado atuante, onde diferenciar-se dos outros por ofertar preços mais baixos. 4 - Imagem: A marca é considerada um dos critérios preponderante na escolha dos seus produtos (bens), onde o processo de criação de valor para o cliente desenvolve-se através da propaganda e comunicação da marca.

Corrêa $(2009$, p. 418) diz que a estratégia determina as operações internas, como o foco principal da gestão nas empresas de serviço, ou seja, as operações possuem características em absorver mais gastos dos recursos físicos, onde as decisões quando tomadas, são difíceis de reverter, por apresentar um processo muito expressivo, onde as estratégias de serviços definidas terão impacto na competitividade no mercado. Para Fitzsimmons e Fitzsimmons (2014), o foco da estratégia de serviço é suprir as necessidades não aten- didas dos clientes. Segundo Lovelock e Wright (2001, p. 187) a estratégia de serviços é a "razão de ser" da empresa, cujo principal objetivo é fornecer valor ao cliente, ou seja, transparecer para o cliente um serviço de excelência como algo irresistível.

Para tanto, Zeithaml (2011) destacam que a estratégia de serviço tem como foco principal o cliente. O grande diferencial competitivo para os clientes está apresentado no serviço ofertado, no entanto, a estratégia está direcionada as percepções do valor percebido e se o cliente observa este valor através da qualidade dos serviços recebidos sobre os fatores pessoais e situacionais. Segundo Johnston \& Clark (2010) estratégia de serviços é desenvolvida por cinco elementos: a elaboração de objetivos corporativos, o ambiente, a elaboração de um conceito de serviço, a identificação de metas para o desempenho operacional e o desenvolvimento de operações. A elaboração de objetivos aprovisiona as metas e os objetivos para a elaboração da estratégia. $\mathrm{O}$ ambiente é o contexto para entendimento das reações de outras empresas e dos clientes. Para tanto, Teboul (1999) a estratégia de segmentação, padronização ou múltiplos apresenta muita importância no resultado esperado pela organização, no entanto, a intensidade da operacionalização do serviço e a interação entre organização e cliente tornam a estratégia de serviço mais admirável.

O mundo dos negócios exige, além do conhecimento comercial tradicional, aumentar a competência dos funcionários na satisfação do cliente, qualidade do serviço e atendimento ao cliente - habilidades essenciais para sustentar a base de clientes existente (HOFFMAN, 2002). A estratégia de serviços se divide em estágios de competitividade operacional, seguindo uma sequência, 1 - disponibilidade para o serviço, 2 - diarista, 3 - competência diferenciada e 4 - excelência em serviços, ou seja, a organização somente completará o ciclo da estratégia de serviço quando conseguir executar de forma plausível todas as etapas dos estágios determinadas no modelo (HOFFMAN, 2009). Já Nóbrega (1997) propõe que, mediante a primeira etapa de execução do modelo de estratégia de serviço seja previamente planejada, delimitando desta forma, o campo de atuação e delimitando o perímetro do mercado a ser atingido, priorizando as oportunidade e necessidade de adoção de uma estratégia de serviço clara que consiga atender o pensamento que cultue o serviço ao cliente. Diante das abordagens dos autores quanto à estratégia de serviços Nóbrega (2013) sugere um modelo adaptado Osterwalder e Pigneur (2010) conforme Quadro 1. 
QUADRO 1: ORIENTAÇÕES PARA FORMULAÇÃO DA ESTRATÉGIA DE SERVIÇO

\begin{tabular}{|c|c|c|c|c|}
\hline OPORTUNIDADES & $\begin{array}{l}\text { COMPETÊNCIAS } \\
\text { ESSENCIAIS }\end{array}$ & $\begin{array}{l}\text { PROMESSA DE } \\
\text { SERVIÇO }\end{array}$ & SERVIÇOS & $\begin{array}{l}\text { SEGMENTOS DE } \\
\text { CLIENTES }\end{array}$ \\
\hline $\begin{array}{l}\text { - Oportunidades } \\
\text { existentes } \\
\text { - Lacunas não } \\
\text { preenchidas } \\
\text { - Serviços a melhorar }\end{array}$ & $\begin{array}{l}\text { - O que sabemos fazer } \\
\text { bem? } \\
\text { - O que gostamos de } \\
\text { fazer? } \\
\text { - O que sabemos e que } \\
\text { a concorrência não } \\
\text { sabe? }\end{array}$ & $\begin{array}{l}\text { - Que valor } \\
\text { estaremos } \\
\text { entregando a } \\
\text { nossos clientes? } \\
\text { Qual benefício o } \\
\text { cliente percebe } \\
\text { de nós? }\end{array}$ & $\begin{array}{l}\text { - Que valor } \\
\text { estaremos } \\
\text { entregando a } \\
\text { nossos clientes? } \\
\text { Qual benefício o } \\
\text { cliente percebe } \\
\text { de nós? }\end{array}$ & $\begin{array}{l}\text { - Quem são nossos } \\
\text { clientes? } \\
\text { - Como estão } \\
\text { segmentados? } \\
\text { - Os serviços são } \\
\text { segmentados por } \\
\text { tipo de clientes? }\end{array}$ \\
\hline CONCORRENTES & DIFERENCIAIS & $\begin{array}{l}\text { POR QUE ESCOLHER } \\
\text { MINHA EMPRESA }\end{array}$ & SERVIÇOS & $\begin{array}{l}\text { SEGMENTOS DE } \\
\text { CLIENTES }\end{array}$ \\
\hline $\begin{array}{l}\text { Que concorrentes } \\
\text { mais se aproximam } \\
\text { do nosso perfil de }\end{array}$ & $\begin{array}{l}\text { - Em que nos } \\
\text { diferenciamos dos }\end{array}$ & $\begin{array}{l}\text { - Por que os } \\
\text { clientes deveriam } \\
\text { nos escolher para }\end{array}$ & Diferenciação & Custo \\
\hline atuação? & diretos? & $\begin{array}{l}\text { os serviços que } \\
\text { deseja? }\end{array}$ & \multicolumn{2}{|c|}{ Enfoque } \\
\hline
\end{tabular}

Fonte: (NÓBREGA, 2013, p. 85) adaptado de (OSTERWALDER; PIGNEUR, 2010)

Para Porter (2005) existem três estratégias genéricas, que poderão ser utilizadas em conjunto ou isoladamente na tomada de decisão. Em outras palavras, o autor, se refere ao posicionamento estratégico em que a organização se propõe ou pretende ser visto pelo consumidor. No âmbito de toda indústria, o autor sugere as estratégias de posicionamento: liderança em custo e diferenciação.

Todas as estratégias traçadas pelas organizações devem ser claras e obrigatórias, porém, não são todas as empresas que determinam estratégias como obrigatória. Segundo Berry (1995) e Lovelock (2001) as organizações servidoras têm uma estratégia de servi- ços clara e obrigatória, para tanto, essas organizações possuem uma "razão de ser"e, ou seja, missão que inspira, energiza a organização de tal forma, que representa a palavra serviço.

Os serviços desenvolvidos por meio da tecnológica (internet) indicam um futuro promissor, bem como, as novas formas de internacionalização que permite a globalização a partir do estabelecimento de uma organização (GRÖNROOS, 2016).

Entretanto, para atuar num mercado de segmentação particular, o autor sugere a estratégia e posicionamento enfoque, conforme Quadro 2 abaixo:

\section{QUADRO 2: ESTRATÉGIAS GENÉRICAS DE PORTER - POSICIONAMENTO}

\begin{tabular}{|c|c|c|c|}
\hline $\begin{array}{l}\text { ESTRATÉGIA } \\
\text { GENÉRICA }\end{array}$ & CARACTERISTICAS & VANTAGENS & DESVANTAGENS \\
\hline $\begin{array}{l}\text { Liderança em } \\
\text { Custo }\end{array}$ & $\begin{array}{l}\text { Grande penetração no } \\
\text { mercado através dos preços } \\
\text { baixos que os concorrentes. }\end{array}$ & $\begin{array}{l}\text { Os custos são mais baixos; } \\
\text { Linha de produtos básicos. }\end{array}$ & $\begin{array}{l}\text { Pouca variedade, os produtos são } \\
\text { constituídos em massa. }\end{array}$ \\
\hline Diferenciação & $\begin{array}{l}\text { Apresenta penetração no } \\
\text { mercado pela diversificação } \\
\text { dos bens e serviços, } \\
\text { algo diferenciado dos } \\
\text { concorrentes. }\end{array}$ & $\begin{array}{l}\text { Variações de produtos com } \\
\text { ênfase na diferenciação. }\end{array}$ & Preços elevados \\
\hline Enfoque & $\begin{array}{l}\text { Possui alvo principal os } \\
\text { "nichos de mercados" } \\
\text { personalizados com alto } \\
\text { padrão. }\end{array}$ & $\begin{array}{l}\text { Oferta de bens e serviços } \\
\text { individualizados ou } \\
\text { personalizados. }\end{array}$ & Preços mais elevados \\
\hline
\end{tabular}

Fonte: Adaptado de Porter (2005). 
QUADRO 3: UNIDADES TEMÁTICAS DE COMPARAÇÃO ENTRE OS MODELOS

\begin{tabular}{|l|c|c|c|c|c|c|c|c|c|}
\hline DIMENSÃO & A1 & A2 & A3 & A4 & A5 & A6 & A7 & A8 & A9 \\
Conceituação & I/S & I/S & I/S & I/S & I/S & I/S & I/S & I/S & I/S \\
Modelos \& Perspectivas & I/S & I/S & I/S & I/S & I/S & I/S & I/S & I/S & I/S \\
Posicionamento & I/S & I/S & I/S & I/S & I/S & I/S & I/S & I/S & I/S \\
Estratégia de Preço & $\mathrm{L}$ & $\mathrm{L}$ & I/S & $\mathrm{L}$ & I/S & I/S & $\mathrm{L}$ & $\mathrm{L}$ & $\mathrm{I} / \mathrm{S}$ \\
Orientação para Serviços & $\mathrm{L}$ & $\mathrm{L}$ & $\mathrm{I} / \mathrm{S}$ & $\mathrm{L}$ & $\mathrm{L}$ & $\mathrm{L}$ & $\mathrm{I} / \mathrm{S}$ & $\mathrm{L}$ & $\mathrm{L}$ \\
\hline
\end{tabular}
Legenda: A1 (Corrêa); A2 (Fitzsimmons \&Fitzsimmons), A3 (Grönroos), A4 (Hoffman), A5 (Johnston
\& Clark), A6 (Lovelock), A7 (Nóbrega), A8 (Teboul) e A9 (ZeithamI)

De modo geral, esta construção foi extraída a partir da abordagem de cada autor, e serviu de orientação para o aprofundamento do comparativo, que será mostrado, em detalhe, na seção de resultados do presente artigo.

\section{METODOLOGIA}

O método desenvolvido para a pesquisa é um estudo teórico que tem como objetivo identificar interfaces entre os principais modelos teóricos sobre a estratégia de serviços. $\mathrm{O}$ esquema metodológico da pesquisa está representado no Figura 1.

FIGURA 1: ESQUEMA METODOLÓGICO

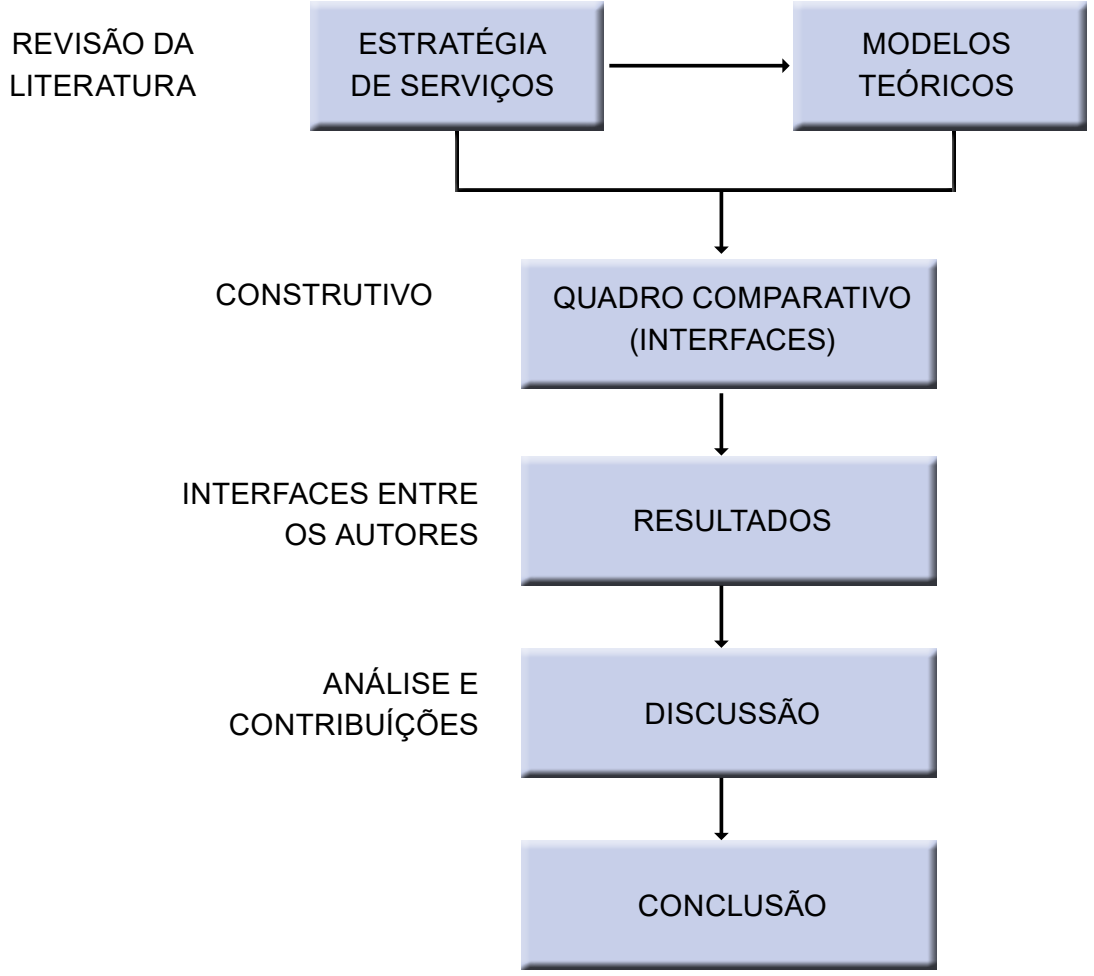

Fonte: Elaboração própria 
As variáveis analíticas foram extraídas da literatura e incorporadas no quadro comparativo das interfaces, com objetivo de identificar as interfaces, similaridades e lacunas. Diante do estudo realizado percebeu-se as características que foram mais referenciados pelos autores, mediante o construto estratégia de serviços. Para tanto, optou-se por dividir em etapas denominadas de dimensões, que foram atribuídas por letras a) conceituação, b) modelos e perspectivas, c) posicionamento, d) estratégia de preço e por fim, e) orientada para serviço.

Desta forma, foram estudados na literatura os principais autores sobre a estratégia de serviços Nóbrega (2013), Grönroos (2009) e Fitzsimmons \& Fitzsimmons (2014), Hoffman (2009), Johnston \& Clark (2010), Lovelock (2002), Teboul (1999), Zeithaml (2011), Corrêa (2009), logo, foram identificadas as interfaces, similaridades e lacunas existentes entre os modelos. Os autores foram selecionados mediante a relevância durante a triagem dos artigos na base de dados, bem como, o maior número de citações desses autores na base teórica.

Os artigos foram coletados na base de dados da Emerald Insight, utilizando os seguintes critérios: 1 - utilizaram-se as palavras-chaves estratégia de serviços, "serviços", strategy Service e marketing de serviço; 2 - o período selecionado para coleta dos artigos foi entre 2006 a 2016, porém, utilizando como critério a relevância. Os dados foram inseridos nas colunas referente a cada citação dos autores e dimensões previamente determinadas. Os autores que não apresentaram citação quanto à dimensão estabelecida foram identificados no quadro comparativo com um "x".

Após a seleção dos artigos foram realizadas as leituras buscando identificar as interfaces, similaridades e lacunas. Para representar de forma clara e simplificada, foi elaborado um quadro que representasse as interfaces, similaridades e lacunas entre os principais modelos de estratégia de serviços. As lacunas estão identificadas por um " $x$ " no quadro de análise

De posse dos principais construtos teóricos sobre a estratégia de serviços foi possível observar as interfaces e similaridades existentes entre os modelos teóricos de cada autor, no entanto, desenvolveu-se um quadro sobre as interfaces entre os principais modelos da estratégia de serviços. Segundo Flick (2004) investigar teorias, fatos, coisas ou pessoas, expondo suas diferenças e semelhanças.

\section{RESULTADOS E DISCUSSÃO}

Esta seção apresenta e discute os principais resultados alcançados no estudo sobre as interfaces identificadas nos modelos teóricos sobre a estratégia de serviços estudados. Primeiramente são apresentados os resultados, que correspondem às interfaces, similaridades e lacunas entre cada abordagem estudada. Em seguida é feita a discussão dos resultados apresentados.

\section{1 RESULTADOS}

Para exposição das interfaces entre os construtos destacados na literatura sobre as perspectivas da estratégia de serviços. Diante disso, foi possível construir um quadro comparativo apresentando as interfaces, similaridades e lacunas existentes nos principais modelos teóricos. Diante disso, estabeleceu-se como critério de análise o método comparativo entre as perspectivas das abordagens dos modelos teóricos. Assim, o objetivo do Quadro 4 foi facilitar o entendimento e observações entre as interfaces, similaridades e lacunas denominadas entre as teorias estudadas.

Para os assuntos em que a abordagem do autor não trouxe contribuições, identificados como lacunas, identificou-se pela letra "X".

Quadro 4: Interfaces entre os principais modelos teóricos sobre Estratégia de Serviços 
QUADRO 2: ESTRATÉGIAS GENÉRICAS DE PORTER - POSICIONAMENTO

\begin{tabular}{|c|c|c|c|c|c|c|c|c|c|}
\hline DIMENSÃO & CORRÊA & FITZSIMMONS & GRÖNROOS & HOFFMAN & JOHNSTON & LOVELOCK & NÓBREGA & TEBOUL & ZEITHAML \\
\hline Conceituação & $\begin{array}{l}\text { a) Estratégia } \\
\text { corporativa; } \\
\text { Estratégia } \\
\text { competitiva; } \\
\text { Estratégia de } \\
\text { operações } \\
\text { (prescrita e } \\
\text { resultante) } \\
\text { e Estratégia } \\
\text { emergente; }\end{array}$ & $\begin{array}{l}\text { a) Estratégia } \\
\text { Operacional; } \\
\text { Estratégias } \\
\text { competitivas em } \\
\text { serviços; } \\
\text { serviços podem } \\
\text { ser copiados, } \\
\text { poder de } \\
\text { negociação, } \\
\text { sazonalidade } \\
\text { da demanda e } \\
\text { fidelizar clientes; }\end{array}$ & $\begin{array}{l}\text { a) É a junção entre } \\
\text { os conceitos de } \\
\text { serviços com a } \\
\text { missão e estratégia } \\
\text { do negócio, } \\
\text { estabelecendo } \\
\text { desta forma o que } \\
\text { deverá ser feito, } \\
\text { para quem, como e } \\
\text { com quais recursos } \\
\text { e benefícios devem } \\
\text { ser ofertados }\end{array}$ & $\begin{array}{l}\text { a) Estágios de } \\
\text { competitividade } \\
\text { operacional: } \\
1 \text { - disponível } \\
\text { para o serviço. } \\
\text { 2. diarista. } 3 . \\
\text { competência } \\
\text { diferenciada e } 4 \text {. } \\
\text { Excelência em } \\
\text { serviços. }\end{array}$ & $\begin{array}{l}\text { a) Estratégia } \\
\text { envolvendo } \\
5 \text { elementos } \\
\text { (objetos } \\
\text { corporativos, } \\
\text { ambiente, } \\
\text { conceito, } \\
\text { objetivos de } \\
\text { desempenho } \\
\text { e operação) } \\
\text { e Estratégias } \\
\text { planejadas ou } \\
\text { emergentes. }\end{array}$ & $\begin{array}{l}\text { a) atrair clientes } \\
\text { de outros } \\
\text { segmentos, no } \\
\text { lugar, tempo e } \\
\text { preço certo; }\end{array}$ & $\begin{array}{l}\text { a) Estratégia } \\
\text { corporativa, } \\
\text { competitiva e } \\
\text { operações. } \\
\text { Estabelece como } \\
\text { a organização } \\
\text { propõe a } \\
\text { oferecer serviços } \\
\text { aos clientes, } \\
\text { agregando valor } \\
\text { ao seu produto } \\
\text { principal, seja um } \\
\text { bem ou serviço. }\end{array}$ & $\begin{array}{l}\text { a) Estratégia de } \\
\text { segmentação de } \\
\text { padronização } \\
\text { ou múltiplos: } \\
\text { importância } \\
\text { do resultado, } \\
\text { intensidade } \\
\text { do serviço e } \\
\text { interação; }\end{array}$ & $\begin{array}{l}\text { a) Estratégia } \\
\text { de precificação: } \\
\text { tem como foco } \\
\text { principal o } \\
\text { conhecimento } \\
\text { do cliente } \\
\text { sobre preço do } \\
\text { serviço; }\end{array}$ \\
\hline $\begin{array}{l}\text { Modelos, } \\
\text { perspectivas }\end{array}$ & $\begin{array}{l}\text { b) Modelo top- } \\
\text { down: estratégia } \\
\text { corporativa, } \\
\text { competitiva } \\
\text { e funcional } \\
\text { (marketing, } \\
\text { operações e } \\
\text { finanças); }\end{array}$ & $\begin{array}{l}\text { b) Estratégia } \\
\text { competitiva: } \\
\text { liderança } \\
\text { em custos, } \\
\text { diferenciação e } \\
\text { focalização; }\end{array}$ & $\begin{array}{l}\text { b) Perspectiva de } \\
\text { serviço, produto } \\
\text { central, preço e } \\
\text { imagem; }\end{array}$ & $\begin{array}{l}\text { b) Discriminação } \\
\text { de preços: prática } \\
\text { de cobrar preços } \\
\text { diferentes, } \\
\text { de clientes } \\
\text { diferentes, para o } \\
\text { mesmo serviço; }\end{array}$ & $\begin{array}{l}\text { b) Conhecendo } \\
\text { competências } \\
\text { essenciais de } \\
\text { operação x } \\
\text { gerentes x novos } \\
\text { conceitos de } \\
\text { serviços; }\end{array}$ & $\begin{array}{l}\text { b) Segmentar } \\
\text { clientela e } \\
\text { informar caráter } \\
\text { específico do } \\
\text { serviço; }\end{array}$ & $\begin{array}{l}\text { b) Componentes } \\
\text { da estratégia } \\
\text { de serviços: } \\
\text { oportunidades; } \\
\text { competências } \\
\text { essenciais; } \\
\text { promessa do } \\
\text { serviço; serviços; } \\
\text { segmentos } \\
\text { de clientes; } \\
\text { concorrentes; } \\
\text { diferenciais; } \\
\text { porque escolher } \\
\text { minha empresa e } \\
\text { posicionamento }\end{array}$ & $\begin{array}{l}\text { b) Segmentação: } \\
\text { padronizados, } \\
\text { personalizados } \\
\text { ou múltiplos; }\end{array}$ & $\begin{array}{l}\text { b) Preço como } \\
\text { indicador de } \\
\text { qualidade: } \\
\text { depende de } \\
\text { alguns fatores; }\end{array}$ \\
\hline $\begin{array}{l}\text { Posicionamento } \\
\text { Estratégia de } \\
\text { preço }\end{array}$ & $\begin{array}{l}\text { c) Estratégia } \\
\text { de operações. } \\
\text { Desempenho: } \\
\text { acesso, } \\
\text { velocidade, } \\
\text { consistência, } \\
\text { atendimento, } \\
\text { flexibilidade, } \\
\text { conforto e } \\
\text { qualidade } \\
\text { (satisfação) Áreas } \\
\text { de decisão: quais } \\
\text { ações sobre quais } \\
\text { recursos e para } \\
\text { que; }\end{array}$ & $\begin{array}{l}\text { c) Conquistando } \\
\text { clientes: } \\
\text { disponibilidade, } \\
\text { conveniência, } \\
\text { confiabilidade, } \\
\text { preço, } \\
\text { personalização, } \\
\text { qualidade, } \\
\text { reputação, } \\
\text { segurança e } \\
\text { rapidez; }\end{array}$ & $\begin{array}{l}\text { c) Estratégia de } \\
\text { relacionamento: } \\
\text { contato direto com } \\
\text { cliente, banco de } \\
\text { dados, serviço } \\
\text { orientado para o } \\
\text { cliente, redefinir } \\
\text { processos e } \\
\text { estabelecer } \\
\text { parcerias; }\end{array}$ & $\begin{array}{l}\text { c) Considerações } \\
\text { de custo: preço } \\
\text { não calculado } \\
\text { antes de serem } \\
\text { concluídos; }\end{array}$ & $\begin{array}{l}\text { c) Elementos } \\
\text { chaves: objetivos } \\
\text { corporativos, } \\
\text { ambiente, } \\
\text { conceito, } \\
\text { objetivos de } \\
\text { desempenho e } \\
\text { operação; }\end{array}$ & $\begin{array}{l}\text { c) Estratégia de } \\
\text { posicionamento: } \\
\text { atributos, preço, } \\
\text { qualidade, } \\
\text { concorrentes, } \\
\text { ocasiões de } \\
\text { uso, usuário } \\
\text { e classe do } \\
\text { produto; }\end{array}$ & $\begin{array}{l}\text { c) Oportunidades, } \\
\text { concorrentes, } \\
\text { competências } \\
\text { individuais, } \\
\text { diferenciais, o } \\
\text { que pretende } \\
\text { oferecer, } \\
\text { importância e } \\
\text { desempenho, } \\
\text { serviços } \\
\text { oferecidos e } \\
\text { segmentar } \\
\text { clientes; }\end{array}$ & $\begin{array}{l}\text { c) Proposta de } \\
\text { valor: resultados } \\
\text { ampliados, } \\
\text { interação do } \\
\text { processo, } \\
\text { interação de } \\
\text { funcionários, } \\
\text { credibilidade e } \\
\text { preço; }\end{array}$ & $\begin{array}{l}\text { c) Estratégia de } \\
\text { precificação: } \\
\text { valor é preço } \\
\text { baixo, valor é } \\
\text { tudo que eu } \\
\text { quero em um } \\
\text { serviço e valor } \\
\text { é a qualidade } \\
\text { que obtenho } \\
\text { pelo preço que } \\
\text { pago; }\end{array}$ \\
\hline \multirow[t]{2}{*}{$\begin{array}{l}\text { Orientação } \\
\text { para serviço }\end{array}$} & $X$ & $X$ & $\begin{array}{l}\text { d) Reduzir preços, } \\
\text { custos, aumentar } \\
\text { verbas publicitárias } \\
\text { e fortalecer } \\
\text { desenvolvimento } \\
\text { de produtos; }\end{array}$ & $X$ & $\begin{array}{l}\text { d) Decisões de } \\
\text { preço: maximizar } \\
\text { os benefícios } \\
\text { e minimizar os } \\
\text { custos para } \\
\text { o cliente e } \\
\text { minimizar custo } \\
\text { para organização; }\end{array}$ & $\begin{array}{l}\text { d) Estratégia } \\
\text { de preços: } \\
\text { base no custo, } \\
\text { concorrência e } \\
\text { no valor; }\end{array}$ & $X$ & $X$ & $\begin{array}{l}\text { d) Preço } \\
\text { justo: esforço } \\
\text { de melhoria } \\
\text { produz } \\
\text { aumento } \\
\text { do nível de } \\
\text { satisfação; }\end{array}$ \\
\hline & $X$ & $X$ & $\begin{array}{l}\text { e) Estratégia } \\
\text { orientada para } \\
\text { o serviço: } \\
\text { interação, melhor } \\
\text { qualidade, imagem } \\
\text { corporativa, mais } \\
\text { volume de vendas; }\end{array}$ & $X$ & $X$ & $X$ & $\begin{array}{l}\text { As empresas } \\
\text { adotam } \\
\text { estratégias de } \\
\text { serviços para } \\
\text { "servir bem" }\end{array}$ & $X$ & $X$ \\
\hline
\end{tabular}

Fonte: Elaboração própria, 2016. Adaptado de Nóbrega (2013), Grönroos (2009), Fitzsimmons \& Fitzsimmons (2014), Hoffman (2009), Johnston \& Clark (2010), Lovelock (2002), Teboul (1999), Zeithaml (2011) e Corrêa (2009). 


\subsection{DISCUSSÃO}

As perspectivas sobre a estratégia de serviços dos principais modelos teóricos foram divididas em dimensões para facilitar o entendimento das interfaces, similaridade e lacunas existentes nos construtos estudados. Diante das análises realizadas foi possível perceber que apresentaram mais interfaces e similaridades entre os elementos abordados na literatura do que lacunas. Para tanto, dividiu-se em etapas denominadas de dimensões atribuídas por letras a) conceituação, b) modelos e perspectivas, c) posicionamento, d) estratégia de preço e por fim, e) orientada para serviço.

Pode-se perceber que, no item Conceituação foi possível evidenciar interfaces entre os construtos teóricos sobre a estratégia de serviços não apresentando nenhuma lacuna. As similaridades identificadas nessa dimensão mostram mais congruência entre Teboul (1999), Grönroos (2009), Hoffman (2009), Johnston e Clark (2010) e Nóbrega (2013), os autores destacam a estratégia de serviços orientada para serviços. Os demais autores abordam a estratégia de serviço a orientação para serviços, bem como, observando alguns atributos como preço e demanda. As similaridades encontradas nesta dimensão foram: os conceitos identificados de cada autor, bem como, as semelhanças apresentadas quanto a definição da estratégia de serviços. Todos os autores citados apresentam modelos teóricos pertinentes para ampliação da fundamentação teórica acerca da estratégia de serviços.

Portanto, com base nos resultados obtidos nesta pesquisa teórica, a conceituação atribuída para estratégia de serviços estabelece uma relação entre o que a organização se propõe a oferecer (bens ou serviços) agregando valor para o cliente. As interfaces e similaridades identificadas entre os autores apontam para uma consistência sólida em relação a conceituação da estratégia de serviços. As organizações estão evoluindo para um ponto central nos negócios, a proposta primordial é servir atendendo as necessidades dos clientes, bem como, tornar o cliente parte superior na hierarquia da empresa, essa é a razão para existência das organizações (FISK, 2011).

Com relação à dimensão modelos \& perspectivas, os modelos teóricos estudados apresentam congruência e similaridades, não sendo evidenciada nenhuma lacuna. Identificou-se que estratégias competitivas relatadas por Fitzsimmons \& Fitzsimmons (2014), as perspectivas de Grönroos (2009), as estratégias genéricas citadas por Nóbrega (2013) e o modelo top down de Corrêa (2009), indicam harmonia nas interfaces encontradas. Os demais autores citam as estratégias de serviços de forma similares em função da perspectiva de cada um. No entanto, Nóbrega (2013) descreve as etapas dos componentes estratégicos que deverão ser analisadas para traçar um modelo de estratégia de serviços conforme Quadro 1 descrito no referencial teórico, esse modelo foi adaptado de Osterwalder \& Pigneur (2010) em seu estudo sobre as modelagens de negócios utilizando o "canvas" como ferramenta, onde a proposta de valor inclui o pacote de serviços que cria valor para cada segmento específico de cliente.

Portanto, os resultados extraídos durante a pesquisa mostram que os modelos e perspectivas teóricos estudados apresentam congruência e harmonia entre os autores. Pode-se concluir que, os modelos teóricos propostos são consolidados podendo ser adaptado pelas organizações que buscam soluções gerenciais para os negócios, por meio da estratégia de serviços. Corroborando Larentis et al. (2013) afirma que, as empresas que apresentam modelos que tenham relacionamento com os clientes de forma positiva por meio da prestação dos serviços, troca de informações, conhecimento do seu eixo de atuação aplicando estratégia de serviços poderão trazer resultados na melhoria dos serviços.

$\mathrm{Na}$ dimensão posicionamento, os autores mencionam o posicionamento no mercado de atuação, onde foi possível perceber as interfaces entre os modelos de Fitzsimmons \& Fitzsimmons (2014), de Grönroos (2009), Nóbrega (2013), Lovelock (2002) e Zeithaml (2011): as interfaces encontradas foram as estratégias de posicionamento por meio das variáveis identificadas pelos autores. No entanto, os demais modelos teóricos apresentam similaridades sobre a perspectiva posicionamento, são elas: as estratégias de operações (posicionamento) que são elementos ou variáveis chaves para conquistar os clientes, bem como, as oportunidades, concorrências e competências são aspectos importantes para definição da proposta de valor da organização. Segundo Porter (2005), o posicionamento é o despertar para o cliente, ou seja, como a empresa pretende ser vista pelo olhar do cliente, na perspectiva de diferenciação, liderança em preço (preços baixos) ou enfoque (nichos de mercado específicos). Corroborando Pacheco (2016) as perspectivas das organizações, quanto aos serviços prestados estão direcionadas à percepção do cliente na verificação do atendimento aos requisitos (expectativas) com ausência de falhas.

Portanto, em termos de Posicionamento os resultados mostram que os autores relatam sobre o merca- 
do de atuação. Definir a estratégia de posicionamento mais adequada para organização requer estudo do ambiente na qual a organização está inserida, bem como, analisar as vantagens e competitividade dos negócios. Corroborando Murti, Deshpande e Srivastava (2013) a alta qualidade dos serviços entregue aos clientes oferece uma grande oportunidade para às organizações de se diferenciar estrategicamente dos seus oponentes, bem como, obter maior vantagem competitiva por meio das estratégias de serviços adotadas.

Já na dimensão estratégia de preço, que visa proporcionar aos serviços os valores justos pagos pelos clientes diante dos serviços recebidos. Os autores Grönroos (2009), Johnston \& Clark (2010), Lovelock (2002) e Zeithaml (2011) apresentam interfaces que são: a definição do preço, as decisões quanto aos preços estabelecidos pelos bens ou serviços, bem como, a definição do preço justo. Lacunas foram identificadas nos modelos representados pelos demais autores Corrêa (2009), Fitzsimmons \& Fitzsimmons (2014), Hoffman (2009), Nóbrega (2013) e Teboul (1999), que não apresentam definições sobre estratégia de preço.

Portanto, pode-se concluir que a estratégia de preço ainda apresenta lacunas, ou seja, apresenta necessidade de mais pesquisas a respeito do assunto. As lacunas existentes sobre a temática indicam que há um campo vasto de pesquisa para ser explorado. Corroborando Johnston \& Clark (2010) A decisão da estratégia de preço maximiza os benefícios, minimizando os custos tanto para o cliente quanto para a organização.

Sobre a dimensão orientação para serviço, apenas Grönroos (2009) menciona estratégia orientação para o serviço, onde a interação apresenta uma melhoria na qualidade dos serviços ofertados através da imagem corporativa, aumentando, desta forma, o volume de vendas. Portanto, não foi possível identificar interfaces e similaridades, uma vez que os demais autores não apresentam definições sobre a estratégia orientada para o serviço. Corroborando Nóbrega (2013) As empresas adotam estratégias de serviços com o objetivo de "servir bem" para se manterem no mercado. Diante disso, pode-se afirmar que Teboul (1999), Lovelock (2002), Corrêa (2009), Hoffman (2009), Johnston \& Clark (2010), Zeithaml (2011), Fitzsimmons \& Fitzsimmons (2014), apresentam lacunas neste elemento analisado teoricamente. Conclui-se que, baseado nos resultados apresentado nesta pesquisa, a dimensão orientação para serviço apresenta lacunas, que do ponto de vista científico requer mais pesquisas sobre o assunto. As contribuições realizadas pelos autores indicam a base para as próximas pesquisas sobre a orientação para serviço no contexto empresarial. Grönroos (2009) a interação apresenta uma melhoria na qualidade dos serviços ofertados através da imagem corporativa, aumentando desta forma o volume de vendas.

Portanto, as interfaces existentes entre os principais modelos teóricos buscam identificar e sintetizar os pontos culminantes entre os construtos, ou seja, o encontro dessas teorias mostra a riqueza encontrada neste estudo. Para tanto, mostrar com facilidade as perspectivas dessas interfaces foi desafiador, por se tratar de elementos similares que apresentam lacunas, em alguns aspectos analisados que compõem os construtos sobre a estratégia de serviços. Os construtos se comunicam através das dimensões denominadas neste estudo, utilizando-se, desta forma, os elementos associados às perspectivas que geram impacto no desempenho dos serviços prestados, através da estratégia de serviços adotada.

Para finalizar, as interfaces mostram a interseção entre os principais modelos teóricos estudados, haja vista que, as similaridades destacadas comungam dos resultados encontrados através de um estudo teórico subjetivo sobre abordagens da temática discutida neste estudo. Entretanto, pode-se evidenciar que os modelos teóricos estudados apresentam lacunas em algumas dimensões determinadas nesta pesquisa, possibilitando estudos futuros aplicados em campo, buscando evidenciar na prática se realmente há presença das lacunas apresentadas no estudo teórico.

\section{CONCLUSÕES}

Modelos consumam apresentar convergências e divergências. Os resultados apresentados nesta pesquisa revelam que os construtos estudados apresentam interfaces, similaridades e lacunas, mostrando distinções quanto aos modelos teóricos de cada autor, ressaltando-se, assim, as contribuições mais significativas de cada abordagem. Assim, considera-se que o objetivo da pesquisa, de modo geral, foi alcançado. O comparativo entre os modelos teóricos foi sintetizado em um quadro-resumo, a partir do qual as principais conclusões são explicitadas a seguir.

$\mathrm{Na}$ Conceituação as principais interfaces foram as contribuições teóricas dos autores quanto aos conceitos atribuídos para estratégia de serviços. As similaridades encontradas foram: A denominação concedida para o conceito "estratégia de serviços" representa uma sinergia entre os autores. Neste item 
não foram identificadas lacunas. Os autores mais destacados nessa dimensão foram Teboul (1999), Grönroos (2009), Hoffman (2009), Johnston e Clark (2010) e Nóbrega (2013).

Com relação a dimensão Modelos e Perspectivas as principais interfaces encontradas foram: as estratégias competitivas, as perspectivas dos modelos teóricos, as estratégicas genéricas e o modelo top down. As similaridades encontradas foram: os modelos e perspectivas dos autores quanto a prestação de serviços. Neste item não foram identificadas lacunas. Nesta dimensão os autores que obtiveram mais destaque foram: Corrêa (2009), Grönroos (2009), Nóbrega (2013) e Fitzsimmons \& Fitzsimmons (2014).

Sobre a dimensão Posicionamento as principais interfaces foram: as variáveis identificadas pelos autores quanto à estratégia de serviços. As similaridades encontradas foram: as estratégias de posicionamento por meio das variáveis identificadas pelos autores. Não foram identificadas lacunas neste item. Os autores mais destacados nessa dimensão foram Lovelock (2002), Grönroos (2009), Zeithaml (2011), Nóbrega (2013) e Fitzsimmons \& Fitzsimmons (2014).

Quanto à dimensão estratégia de preço as interfaces identificadas foram: análise do preço por meio dos custos, a formulação do preço e definição do preço justo. As principais similaridades encontradas foram: os valores pagos pelos clientes mediante os serviços recebidos. As lacunas foram identificadas na dimensão estratégia de preço por meio da ausência de citação dos autores Teboul (1999), Corrêa (2009), Hoffman (2009), Nóbrega (2013), Fitzsimmons \& Fitzsimmons (2014). Por outro lado, os autores mais destacados nessa dimensão foram Lovelock (2002), Grönroos (2009), Johnston \& Clark (2010) e Zeithaml (2011).

Por fim, não menos importante, abordagem sobre a dimensão orientação para serviço contempla as principais interfaces encontradas na pesquisa, que foram: as ações das organizações são realizadas por meio da orientação para serviço, ou seja, servir ao cliente atendendo suas necessidades ou expectativas. A similaridade encontrada foi a necessidade em servir, por meio da prestação do serviço seja um bem ou serviço. As lacunas foram identificadas por meio da ausência de citação dos autores Teboul (1999), Lovelock (2002), Corrêa (2009), Hoffman (2009), Johns- ton \& Clark (2010), Zeithaml (2011), Nóbrega (2013) e Fitzsimmons \& Fitzsimmons (2014). Entretanto, os autores que obtiveram mais destaque foram Grönroos (2009) e Nóbrega (2013), a orientação para serviço está baseada no que e como a organização pretende "servir" ao cliente.

Como implicações gerenciais, embora este estudo seja teórico, empresas e gestores podem adotar uma estratégia de serviço, se não com todas as dimensões, pelo menos com algumas delas, ainda que de forma parcial. As dimensões conceitos e Modelos \& perspectivas, de cunho mais conceitual, têm caráter mais acadêmico-científico. Entretanto as dimensões posicionamento, estratégia de preço e orientação para serviço podem ser consideradas para fins de estabelecimento de uma estratégia de serviço, seja na fase de planejamento de um novo negócio, seja na fase de ajustes e melhoramentos. Os achados desta pesquisa mostram a necessidade de mais pesquisas quanto às dimensões estratégia de preço e orientação para serviço, pelo fato de apresentar lacunas entre os autores estudados, bem como, mais estudos aplicados no setor de serviços.

Diante desse contexto, a pesquisa desenvolvida neste artigo possui limitações com relação à utilização deste modelo sintético apresentado nos resultados, por se tratar de um modelo apenas teórico. Entretanto, essas limitações poderão ser sanadas em trabalhos futuros, uma vez que, este estudo apresenta-se como inicial, para posterior aplicação no campo destinado à pesquisa. Outra limitação identificada durante a pesquisa foi a falta de acesso à alguns artigos na base de pesquisa utilizada, bem como, o critério da relevância sobre a temática durante a busca a temática em questão. Recomenda-se que seja realizada pesquisa aplicada por segmentos de atuação em serviços, buscando identificar se as interfaces identificadas no modelo teórico serão confirmadas na prática através da aplicação de campo.

Para tanto, espera-se que este estudo possa contribuir para a comunidade acadêmica, bem como, tenha utilidade para pesquisas futuras o meio empresarial. Diante do exposto, acredita-se que este texto utilizado contribua para referência bibliográfica em outras pesquisas, demonstrando desta forma a importância e relevância sobre a temática. 
BERRY, L. L.; PARASURAMAN, A. Serviços de marketing: competindo através da qualidade. 3. ed. São Paulo: Maltese, 1995.

BHAT, M. A. Tourism Service Quality: The Dimension-specific Assessment of SERVQUAL. Global Business Review, v.13, n.2, p.327-337, 2012.

BITNER, M. J. Editorial: The Vision for the Future of the Journal of Service Research (JSR). 174, Journal of Service Research, v.17, n.1, p. 3-4, 2014.

CORRÊA, H. Gestão de Serviços: lucratividade por meio de operações e de satisfação dos clientes. Sao Paulo: Atlas, 2009.

FISK, R. P. Liberating services customers: The 21st century challenge. Innovar, v. 21, n. 42, octubre-diciembre de 2011.

FITZSIMMONS, J.,; FITZSIMMONS, M. Administração de Serviços: operações estratégias e tecnologia de informações. São Paulo: Bookman 2014.

FLICK, U. Introdução à pesquisa qualitativa. 2. ed. Tradução Sandra Regina Nertz. São Paulo: Artmed, 2004

FULLER, M. Building-blocks of strategic management: A multi-dimensional, pan-theoretical taxonomy of business strategies. Management Decision, v. 48, n. 1, p. 5-16, 2010.

GOLDER, P. N., Mitra, D., \& Moorman, C. What Is Quality? An Integrative Framework of Processes and States. Journal of Marketing, v.76, n.4, p. 1-23, 2012.

GRÖNROOS, C. Service management and marketing: customer management in service competition. John Wiley \& Sons, 2007.

. Marketing: Gerenciamento e serviços. Rio de Janeiro: Elsevier 2009.

. Internationalization strategies for services: a retrospective. Journal of Services Marketing, v. 30, n. 2, p. 129-132, 2016.

HOFFMAN, K. D.; BATESON, John. E. G. Essentials of services marketing: Concepts, strategies and cases. South-Western Pub, 2002.

.; IKEDA; AKEMI; CAMPOMAR, Marcos Cortez. Princípios de Marketing de Serviços: Conceitos, estratégias e casos. São Paulo: Cengage Learning, 2009

HOFFMANN, Valmir Emil et al. Strategic resources for sustainable competitive advantage of food trucks/ recursos estratégicos para la ventaja competitiva sostenible en food trucks. Revista alcance (Online), v. 23, n. 3, p. 352, 2016.

IBGE. Pesquisa anual de serviços (PAS). Rio de Janeiro: IBGE, 2016. 42p.

JOHNSTON, R.; CLARK, G. Administração de operações de serviço. São Paulo: Atlas 2010. 
LARENTIS, Fabiano; TONDOLO, Vilmar Antonio Gonçalves; CISLAGHI, Tatiane Pellin; CAMARGO, Maria Emilia. Contribuição dos relacionamentos interorganizacionais às operações de serviços: um estudo de casos múltiplos. Revista Eletrônica de Estratégia \& Negócios, Florianópolis, v. 6, n. 3, set./dez.2013.

LOVELOCK, C., \& WRIGHT, L. Serviços: Marketing e gestão. São Paulo: Saraiva 2001.

.Lauren. Principles of service marketing and management. Prentice Hall, 2002.

MURTI, Arjun; DESHPANDE, Aashish; SRIVASTAVA, Namita. Service Quality, Customer (Patient) Satisfaction and Behavioural Intention in Health Care Services: Exploring the Indian Perspective. Journal of Health Management, v.15, n.1, p. 29-44, 2013.

NÓBREGA, Kleber Cavalcanti. Gestão da qualidade em serviços. (Tese) - Escola Politécnica, Departamento de Engenharia de Produção, Universidade de São Paulo, São Paulo, 1997

. Falando de serviços: Um guia para compreender e melhorar os serviços em empresas e organizações. São Paulo: Atlas 2013.

OSTERWALDER, A., ; PIGNEUR, Y. Business Model Generation - Inovação em Modelos de Negócios: Um manual para visionários, inovadores e revolucionários. Rio de Janeiro: Alta Books 2010.

PARASURAMAN, A.; ZEITHAML, V.e e BERRY, L. "Alternatives scales for measuring service quality: a comparative assessment based on psychometric and diagnostic criteria". Journal of Retailing. v.70, n.3, p.201-230, 1994.

PACHECO, Natália Araújo. Cognitive, emotional, and behavioral reactions to service failures. 2016.

PORTER, M. E. Estratégia Competitiva: técnicas para análise de indústrias e da concorrência. Rio de Janeiro: Elsevier 2005.

SARKAR, S.; COSTA, A. IA. Dynamics of open innovation in the food industry.Trends in the Food. Science \& Technology, v.19, n.11, p.574-580, 2008.

TEBOUL, J. A era dos serviços: uma nova abordagem ao gerenciamento. Rio de Janeiro: Qualitymark Ed, 1999.

ZEITHAML, V. Marketing de serviços: a empresa com foco no cliente. Porto Alegre: Bookman 2011. 